\title{
Sensing Magnetic Field with Light and Nanomechanics
}

\author{
Guoqiang Lan ${ }^{1,2}$, Jun-Yu Ou ${ }^{1}$ and Eric Plum ${ }^{1}$ \\ 1. Optoelectronics Research Centre and Centre for Photonic Metamaterials, University of Southampton, Southampton, SO17 1BJ, UK \\ 2. School of Electronic Engineering, Heilongjiang University, No. 74 Xuefu Road, Harbin 150080, China \\ Authore-mail address: erp@orc.soton.ac.uk
}

\begin{abstract}
We demonstrate an optical magnetic field sensor based on a metamaterial-microcavity. Actuation of the microcavity by the magnetic Lorentz force controls its reflectivity. Such sensors promise microscale spatial, millisecond temporal and microtesla magnetic field resolution. ( 2020 The Author(s)
\end{abstract}

\section{Introduction}

Magnetic field underpins applications from navigation and mineral prospecting to data storage and brain function mapping. Therefore, magnetic field sensors that combine small size with large dynamic range, sensitivity, spatial and temporal resolution are important. Here we demonstrate a novel type of optical magnetic field sensor. It consists of a magnetically actuated microcavity formed by a mirror and a metamaterial. Magnetic field controls the cavity length, and thus the reflectivity of the metadevice.

\section{Results}

The sensor consists of a microcavity formed by a static mirror and a magnetically displaced metamaterial (Fig. 1a). The metamaterial is supported by a flexible bridge actuator of nanoscale thickness and length $L$, which is displaced by the Lorentz force, $\mathbf{F}_{\mathrm{L}}=\mathrm{LI} \times \mathbf{B}$, acting on an electrical current $\mathbf{I}$ flowing along the bridge in the presence of a magnetic field $\mathbf{B}$. Such displacement changes the cavity length and thus the reflectivity of the device. Sensitivity, dynamic range and linearity of the sensor can be optimized by metamaterial design. Our metamaterial has been optimized for a quasi-linear sensor response in the near-infrared at wavelengths around $1500 \mathrm{~nm}$.

The sensor was fabricated by thermal evaporation of 50-nm-thick gold on a $250 \mu \mathrm{m}$ x $250 \mu \mathrm{m}$ silicon nitride membrane of $50 \mathrm{~nm}$ thickness, which is held by a silicon frame. The bridge actuator and the metamaterial (Fig. 1b) were made by structuring the gold-coated membrane by focused ion beam milling. The frame is separated from a mirror by a photoresist spacer with a groove defined by photolithography to allow for free movement of the bridge actuator. Electrical current is applied via terminals at either end of the bridge actuator.
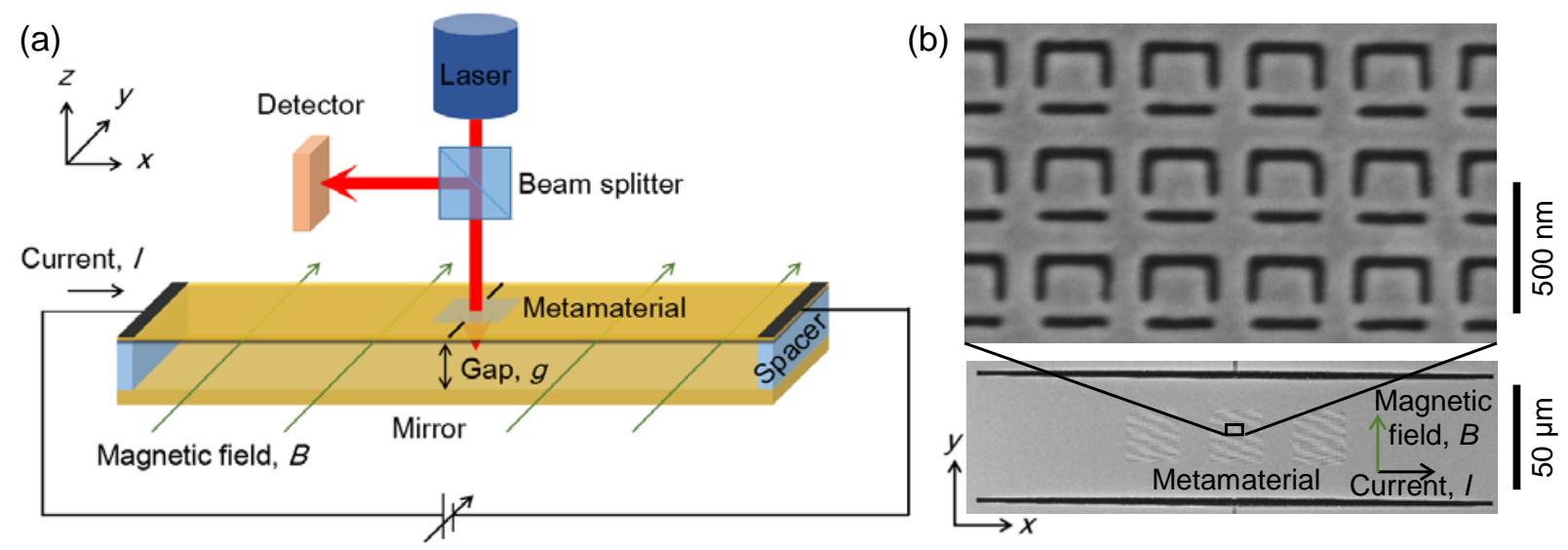

Fig. 1. Optomechanical magnetic field sensor. (a) Schematic of the magnetic field (B) sensor consisting of a Fabry-Pérot microcavity formed by a static mirror and a metamaterial on an elastic beam of length $L$ that carries a current $\mathbf{I}$. The cavity length $g$ is controlled by the magnetic Lorentz force, $\mathbf{F}_{\mathrm{L}}=\mathrm{LI} \times \mathbf{B}$. (b) SEM images of the metamaterial and the supporting elastic beam of $100 \mathrm{~nm}$ thickness. 
Fig. 2 shows the effect of electrical current and magnetic field on the reflectivity spectrum of the sensor. In principle, magnetic displacement via the Lorentz force competes with thermal actuation via resistive heating in such a device. In order to avoid thermal actuation, the device was designed to have a low electrical resistance $(\sim 100 \Omega)$. As magnetic actuation is proportional to electrical current, while thermal actuation depends quadratically on electrical current, a large length/thickness aspect ratio ( 2500) was chosen to maximize magnetic displacement at low-current operation. Indeed, application of electrical current in absence of magnetic field was found to have a negligible effect on the sensor's reflectivity at currents up to $10 \mathrm{~mA}$ (Fig. 2a). In contrast, in the presence of a magnetic field, the reflectivity of the metadevice becomes strongly dependent on electrical current (Fig. 2b). We observe reflectivity changes of up to $25 \%$ at $165 \mathrm{mT}$ magnetic field and $10 \mathrm{~mA}$ current at a range of suitable sensing wavelengths. In this regime, the sensor's reflectivity is linearly dependent on both electrical current and magnetic field. Notably, the dynamic range of the sensor can be flexibly adjusted by changing the applied current. Given that the sensor is still in its linear regime at $165 \mathrm{mT}$ and $10 \mathrm{~mA}$, it will allow measurements of at least $1.65 \mathrm{~T}(10 \mathrm{x}$ larger) at $1 \mathrm{~mA}$ (10x smaller).

In the static regime, assuming reliable detection of reflectivity changes of $0.1 \%$ with a stable laser source and photodetector, the sensor has an accuracy of $\sim 1 \mathrm{mT}$. This will be enhanced considerably by resonant operation of the sensor with a sinusoidally oscillating current at the beam's fundamental resonance frequency of $\sim 6 \mathrm{kHz}$ and lock-in detection of the resulting reflectivity modulation. Numerical modelling predicts two orders of magnitude enhanced beam displacement (and thus reflectivity modulation) at the mechanical resonance, while similar improvements in detection of reflectivity modulation may be expected from detection locked to the oscillation frequency $\omega$. Such detection will reject any thermal effect, as the magnetic field will yield reflectivity modulation at $\omega$, while resistive heating will yield modulation at $2 \omega$. On this basis, we expect sub- $\mu \mathrm{T}$ sensitivity for resonant sensor operation. Considering that mechanical systems can respond to forces that vary more slowly than their fundamental mechanical resonance, we estimate a sub-millisecond response time of the sensor.

(a) Without magnetic field

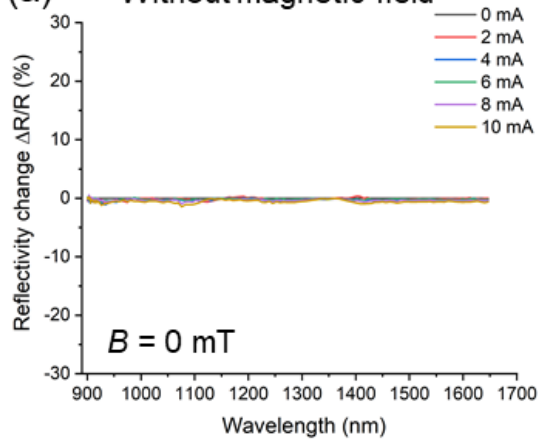

(b) With magnetic field

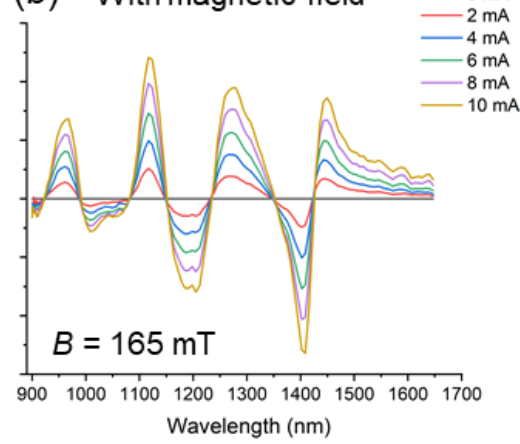

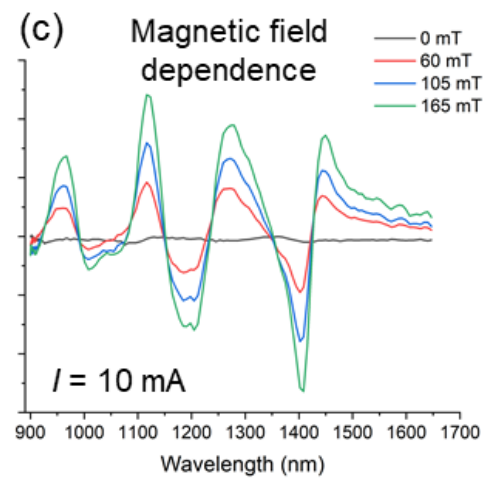

Fig. 2. Magnetic-field-induced reflectivity change. (a) Reflectivity changes due to electrical current are negligible in absence of magnetic field. (b) In the presence of a magnetic field, reflectivity changes linearly with electrical current. (c) At a fixed current, reflectivity changes linearly with the magnetic field.

\section{Summary}

We combine nanomechanics, photonics and metamaterials in order to realize an optical magnetic field sensor of microscale size. Here we provide an experimental proof of the sensing principle. Supported by numerical modelling, we estimate that the sensor has the potential to provide sub-millisecond time resolution and six orders of magnitude of dynamic range. 